\title{
Assessing overexploitation in Mediterranean aquifers using system stability condition analysis
}

\author{
Javier Ibáñez , Jaime Martínez Valderrama , Juan Puigdefábregas \\ Dpto. Estadística y Métodos de Gestión en Agricultura, Universidad Politécnica de Madrid, Spain \\ Estación Experimental de Zonas Áridas, Consejo Superior de Investigaciones Científicas, Spain
}

\begin{abstract}
A B S T R A C T
This paper analyses competitive groundwater exploitation for irrigation by means of a special case of a dynamic predator-prey or human-resource theoretical model. On the human side, the model considers both farmers' rational behaviour and a set of economic parameters. Then the model explicitly links these issues to the dynamics of the natural resource. Equilibrium states are evaluated, and the drivers and conditions for final states leading to groundwater overexploitation are assessed. The theoretical model finally serves to discuss the long-term sustainability of the processes of transformation from traditional to highly intensive pumping-based agricultural systems. These are processes now taking place in aquifers of southern Spain and Maghreb oases.
\end{abstract}

Keywords:

Desertification risk

Alternative long-term states

Groundwater

Oases

Inigation

\section{Groundwater management in arid Mediterranean regions}

The gradual expansion of pumping for irrigation across European countries reached the sunny lands of south-eastern Spain in the second half of the 20th century, as soon as public funds and economies of scale made it so that farmers could afford to acquire the required technology (Martínez Vicente et al., 1989; Tout, 1990). Until then groundwater reserves had remained practically unaltered. First the shortage and then the excessive cost of appropriate soil-drilling techniques had left aquifers in a virgin state.

Thanks to pumping, water is now no longer a constraint on crop yields in these arid regions. Additionally, on account of sand-plot techniques and greenhouses, crops can be grown in a suitable environment, which has largely unshackled production from the surrounding natural conditions. However, a great deal of pesticides and fertilizers has to be used in this highly intensive production system. Also a lot of labour is required, and this has to be cheap to balance the budget. The solution is immigration from African countries, and, because of its proximity, mainly from the Maghreb. In this way, it is possible to harvest selected crop varieties as often as five times a year, and supply the European markets with fresh goods all year round. This is the key to the economic success of the Spanish greenhouses.

A paradigmatic example of this type of agriculture is Campo de Dalías (Almería, Spain), where wastelands have been converted into a field of plastic. This area has grown from the initial 20 experimental hectares to the current figure of 30,000 . As a result, what was one of Spain's poorest 
regions now has the biggest ratio of banks per capita. But, on the other hand, piezometric levels are getting deeper and deeper. This is perceived by farmers in the form of increased pumping costs. Also water salinity is rising, and this narrows the range of alternative crops or even forces them out of some terrains.

The financial impact of groundwater exploitation in Spain has resounded as far as the Maghreb's remote oasis societies. There, traditional water management and distribution laws were carefully established: surface water resources were distributed in times of use depending on availability, and additional water was captured by gravitational force through khettaras, underground tunnels dug into the rock to reach aquifers. However, remittances and knowledge acquired by emigrants are favouring the adoption of pumping, and triggering changes in the traditional systems (Puigdefabregas and Mendizabal, 2004; De Haas, 2001).

Groundwater exploitation in Spain initially followed a competitive strategy, that is to say, farmers owned their wells and pumped water without any agreement with other farmers. However, after symptoms of overexploitation were noticed in different aquifers, irrigation communities have gradually been established, either voluntarily or enforced by the authorities. The aim is to regulate pumping and turn to a social strategy of exploitation. In the Maghreb, the opposite transformation is currently taking place: social systems of aquifer exploitation are being displaced by competitive strategies of pumping, as self-sufficient subsistence farming is transformed into open economy agriculture.

This paper analyses the competitive strategy of groundwater management by means of a set of dynamic equations. The model, which is described in Section 2, is a particular application of the general human-resource model proposed by Ibañez et al. (2008). It serves to formally express the long-term system equilibrium (Section 3) and also to assess the drivers and the conditions for this equilibrium to imply aquifer overexploitation (Section 4). The modelling procedure gives us some keys for theoretically discussing water exploitation in Mediterranean aquifers (Section 5).

\section{A model of the competitive exploitation of groundwater for agricultural production}

As pointed out above, this section particularizes a previously stated human-resource model (Ibañez et al., 2008) to represent the competitive strategy of aquifer exploitation. Throughout the description, capital letters are employed to name variables and small letters to denote parameters.

\subsection{Number of hectares irrigated by pumping}

Let $U$ be the current number of hectares irrigated with water pumped from the aquifer, i.e. not including hectares irrigated by traditional systems in oases. The rate of variation of $U$ is given by

$\frac{\mathrm{dU}}{\mathrm{dt}}=\frac{U_{D}-U}{\text { uat }}$

where $U_{D}$ is the target number of irrigated hectares and 'uat' is the adjustment time.

\subsection{Target number of hectares irrigated by pumping}

First, we assume that there exist a fixed maximum number of arable (i.e. irrigable) hectares denoted by 'umx'. This constraint is due to factors that are exogenous to the model (i.e. geographic or biophysical limitations). Second, only those farmers whose opportunity cost per owned hectare is less than the average per hectare profit, $P_{U}$, would be interested in adopting the pumping-based agricultural production system. For this to be possible there has to be some sort of special rationale driving the farmer's behaviour. Note, however, that the model does not assume that this motivation is there from the very start. The adjustment time 'uat' in Eq. (2.1) would be partially related to the process of a gradual change of mentality, if any. Third, the opportunity cost is assumed to be randomly distributed across the hectares, following an exponential probability distribution, i.e. the greater the opportunity cost, the less likely it is. The target number of irrigated hectares, $U_{D}$, then, is expressed by

$U_{D}=\operatorname{umx}\left\{1-\exp \left[\frac{-\max \left(0, P_{U}\right)}{\operatorname{aoc}}\right]\right\}$,

where 'aoc' is the average per hectare opportunity cost. Note that the term in brackets represents the cumulative distribution function of the exponential random variable opportunity cost particularized to the average per hectare profit $P_{U}$, that is to say, the probability of finding a per hectare opportunity cost that is equal to or less than $P_{U}$. This probability is equal to the fraction of the total area, 'umx', that is the target area at any one time. For example, if the average per hectare profit is greater than the opportunity costs for every hectare, this probability is one, and the target number of hectares is 'umx'. The $\max (\cdot)$ function in Eq. (2.2) assures that the minimum number of target hectares is zero.

\subsection{Production function}

The average per hectare production function is

$\mathrm{Q}_{U}=\operatorname{tch}\left[R_{U}-0.5\left(\frac{R_{U}^{2}}{\mathrm{eqx}}\right)\right]$,

where $R_{U}$ is the average per hectare endowment of water, 'tch' is a technology-related parameter and 'eqx' is the endowment of water allowing maximum production, i.e. $\mathrm{Q}_{U}^{\max }=$ 0.5 tch eqx. Note that endowments of water greater than 'eqx' imply decreasing yields per hectare.

'eqx' could be thought as taking the general form $e_{\mathrm{H}}(1+\Phi)$, $e_{\mathrm{H}}$ being the potential evapotranspiration and $\Phi>0$ a coefficient that could reflect, for example, the excess of water 
needed for soil salt lixiviation. This makes the model compatible with an irrigated area affected by a slight to moderate salinization problem, but never with one where water quality is the main factor determining the limit for agricultural production.

\subsection{Profit per hectare}

The average per hectare profit is given by

$P_{U}=\operatorname{prq} Q_{U}-C_{R} R_{U}-$ fcu,

where 'prq' is the average production price received, $C_{R}$ is the average marginal cost of water and 'fcu' are the average fixed costs per hectare. Given that the cost of lifting water increases with the piezometric depth of the stock of groundwater, which is called $R, C_{R}$ must be a decreasing function of $R$. This is assumed to be exponential:

$C_{R}=\mathrm{cr}_{0} \exp \left(\frac{-R}{\mathrm{pcr}}\right)$

where $\mathrm{Cr}_{0}{ }^{\prime}$ is the maximum cost of pumping, i.e. the cost of getting the last volume of water, $R$ is the stock of groundwater and 'pcr' is a parameter. Note that if Eqs. (2.3) and (2.5) are substituted into Eq. (2.4), $P_{U}$ is a function of $R$ and $R_{U}$.

\subsection{Water demand (endowment) per hectare}

We assume that the owners of the transformed irrigated hectares aim to maximize their own short-term profit, which means that groundwater is exploited competitively. This happens when the value of the marginal product of water is equated with the marginal $\operatorname{cost} C_{R}$, i.e. prq $d Q_{U} / d R_{U}=$ prq tch $\left[1-\left(R_{U} /\right.\right.$ eqx $\left.)\right]=C_{R}$. The farmers follow a hill-climbing heuristic to find the optimal demand for water. This means that they have a target demand for water, $R_{U D}$, which is anchored to current demand, but varies in the economically expected manner under disequilibrium situations (Sterman, 2000). The average values represented in the model would also reflect this process. This can be formally expressed by making

$R_{U D}=R_{U}\left(\frac{\operatorname{prq} \operatorname{tch}\left[1-\left(R_{U} / \text { eqx }\right)\right]}{C_{R}}\right)^{s r r}$

where 'srr' is a parameter. Note that, for example, if the value of the marginal product of water is greater/less than the marginal cost, the target demand $R_{U D}$ will be greater/less than the current demand $R_{U}$ (we avoid repeating "average" before each of the mentioned variables). The parameter 'srr' quantifies the sensitivity of $R_{U D}$ to changes in the relative return to water. The hill-climbing heuristic is completed by making

$\frac{\mathrm{d} R_{U}}{\mathrm{~d} t}=\frac{R_{\mathrm{UD}}-\mathrm{R}_{\mathrm{U}}}{\text { rat }}$

where 'rat' is the adjustment time.

As the water table go deeper with the development of pumping the probability of finding wells where the drawdown becomes excessive could increase, especially in thin aquifers. The rate of extraction descends or even cancels out in those wells, so that the average endowment of water in the area will fall. If such process is likely to occur in the modelled system its effect on $R_{U}$ could be captured by adding to Eq. (2.7) a multiplier ranging from 1 to 0 as the value of $R$ decreases. This possibility is disregarded here.

\subsection{Stock of groundwater}

As a simplifying assumption, groundwater is used for nothing but irrigation. Also, farmers of the pump-irrigated hectares reject water naturally discharged by the aquifer considering it to be a very unpredictable supply. Therefore, the rate of variation of the stock of accessible groundwater $R$ is given by

$\frac{d R}{d t}=r e c-d p 1\left(\frac{R}{r m x}\right)^{d p 2}-(1-r f c) U R_{U}$

The term 'rec' is the average annual recharge, which is related to rainfall, and ' $\mathrm{rfc}$ ' is the fraction of pumped water that percolates back into the aquifer. The second term of Eq. (2.8) is the natural discharge rate. This rate is expressed by a decreasing function of the stock $R$ where ' $r m x$ ' is the maximum aquifer capacity and 'dp1' and 'dp2' are parameters. This formulation allows to freely establishing how fast the natural discharge decay with $R$. Note that it is possible to make natural discharge disappears even at a quite great value of $R$ simply by making 'dp2' high enough.

For the virgin initial conditions (without pumps, or $U=0$ ), the equilibrium of the stock of groundwater, which we will call ' $r$ e', is reached when $\mathrm{rec}=\mathrm{dp} 1(\mathrm{R} / \mathrm{rmx})^{\mathrm{dp} 2}$. In this way, solving for $R$ :

$\mathrm{rne}=\mathrm{rmx}\left(\frac{\mathrm{rec}}{\mathrm{dp} 1}\right)^{1 / \mathrm{dp} 2}$

Under development $(U>0)$, it can be shown that Eq. (2.8) allows reflecting the condition, stated by Bredehoeft (2002), that pumps captures the difference between virgin and equilibrium natural discharges at sustainability.

Finally, the model has a yearly basis. Evaluation of ground water would be done at the end of winter when pumping has been at a minimum for some months. It is assumed the full recovery of the water table (not the water volume) during this period. In this way, local well hydraulics is circumvented in the model.

\section{Long-term equilibrium of the modelled system}

The question of what the final state of an aquifer already involved in or gradually developing a competitive strategy of groundwater exploitation could be explored by analyzing the isoclines of the model described in the last section. As is well known, an isocline defines the points in the phase space where the rate of variation of a state variable equals zero. 


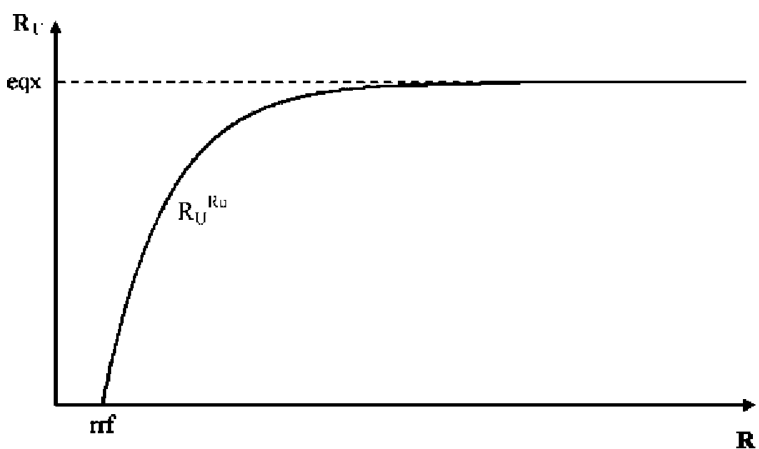

Fig. 1 - The isocline of the per hectare demand for water.

The isocline of the per hectare demand for water $\left(\mathrm{d} R_{U} / \mathrm{dt}=0\right)$, after substituting $C_{R}$ by Eq. (2.5), is ${ }^{1}$

$R_{\mathrm{U}}^{R_{\mathrm{U}}}=\operatorname{eqx} \max \left[1-\frac{\mathrm{cr}_{0}}{\text { prqtch }} \exp \left(\frac{-R}{\mathrm{pcr}}\right)\right]$

The 'max' function is needed to rule out negative demands for water. Note that the expression for per hectare water endowment in equilibrium is the conventional inverted exponential used as the predator functional response in many predator-prey type models (Fig. 1).

For any value of $R$ under 'rrf' there is no demand for water in the equilibrium. Making $R_{U}=0$ in Eq. (3.1) and solving for $R$ we get

$\operatorname{rrf}=-$ pcr $\ln \left(\frac{\text { prq tch }}{\mathrm{cr}_{0}}\right)$.

If prq tch $<\mathrm{cr}_{0}$ then ' $r$ f' is a positive stock of groundwater, which, due to economic and technical constraints, is inaccessible for exploitation (Fig. 1). However, if $\mathrm{prq} t \mathrm{th}>\mathrm{cr}_{0}$, farmers could demand in principle even the last drop of groundwater.

The isocline of the stock of groundwater $(\mathrm{dR} / \mathrm{dt}=0)$, after solving for $U$, is given by

$U^{\mathrm{R}}=\min \left[\mathrm{umx}, \frac{\mathrm{rec}-\mathrm{dp} 1(\mathrm{R} / \mathrm{rmx})^{\mathrm{dp} 2}}{(1-\mathrm{rfc}) \mathrm{R}_{\mathrm{U}}}\right]$.

The ' $m$ in' function is needed because the upper boundary of the number of hectares is always 'umx'. Below this limit, Eq. (3.3) is simply the aquifer's net rate of recharge divided by the net consumption of water per hectare.

Finally, the isocline of the transformed hectares $(\mathrm{dU} / \mathrm{dt}=0)$ is

$U^{U}=U_{D}=u m x\left\{1-\exp \left[\frac{-\max \left(0, P_{U}\right)}{\operatorname{aoc}}\right]\right\}$

The per hectare profit $P_{U}$, which, remember, depends on $R$ and $R_{U}$, is given in Eq. (2.4). The adjustment times needed for the equilibrium of both the groundwater stock and the transformed hectares depend on things like the rate at which an economic rationale for agricultural production spreads, the

\footnotetext{
${ }^{1}$ In the isoclines' notation, the superscript denote the respective state variable. For example, $U^{R}$ is the isocline of $R$, which is eventually solved for $U$.
}

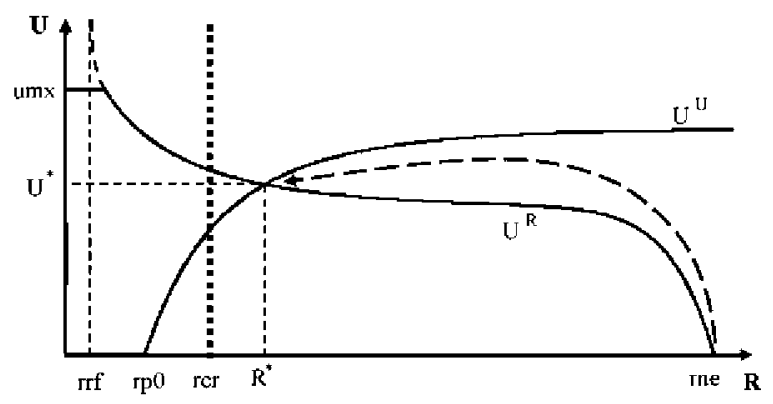

Fig. 2 - An instance of (1) the isoclines of both the transformed hectares $\left(U^{U}\right)$ and the stock of groundwater $\left(U^{R}\right) ;(2)$ the final steady state of the system $\left(R^{*}, U^{*}\right)$, and (3) the trajectory of a complete transformation from a traditional to a competitive system of exploitation (dashed arrow).

time taken to acquire the initial capital, the time required to dig a well and install the irrigation system. These adjustment times are necessarily far greater than it would take farmers already producing (i.e. already having an economic rationale) to adjust their water demands to equilibrium. This would mainly depend on the time it takes to find out the current technical and economic parameter values of interest (e.g. prices, cost). In this way, we can adopt the quasi-steady state assumption (Edelstein-Keshet, 1988) according to which $R_{U}$ can be substituted by its equilibrium value in Eqs. (3.3) and (3.4). Clearly, this substitution results in the isoclines $U^{R}$ and $U^{U}$ being two functions of R. Fig. 2 illustrates the generic form of these two isoclines and the parametric values delimiting them.

The curve $U^{R}$ represents the points in the phase plane $(R$, $U$ ) where there is no variation of water levels and $U^{U}$ those points where there is no variation of the irrigated hectares. Therefore, the steady-state or long-term equilibrium of both $R$ and $U$ is reached simultaneously only at the intersection point $\left(R^{*}, U^{*}\right)$.

The isocline $U^{R}$ obviously intersects with the axis $U=0$ at the virgin volume $R=$ rne (Eq. (2.9)). Given that $R_{U}$ is in the divisor of $U^{R}$, this rises to infinity at rrf. So it is clear that the system equilibrium could never fall below this stock of groundwater. Clearly, too, the intersection of the isocline $U^{U}$ with the axis $U=0$ is the value of the stock of groundwater that makes the per hectare profit exactly zero (after substituting $R_{U}$ by its equilibrium value, $P_{U}$ is just a function of $R$ ). This stock of groundwater has been labelled as $r p_{0}$ in Fig. 2. Finally, note that for $R=\operatorname{rrf}$ or, alternatively, for $R_{U}=0$, the per hectare profit is $P_{U}=-\mathrm{fcu}$ (see Eq. (2.4)). In other words, if no water is employed for irrigation there is no production and no returns, although fixed cost must be paid yet. This means that profit has to be exactly zero before $R$ comes to be equal to rrf. Therefore, for any positive per hectare fixed cost, fcu (i.e. for any aquifer, in fact), it is verified that

$\mathrm{rp}_{0}>\operatorname{rrf}$

That is, the average profit cancels out at a greater stock of groundwater than that making the average per hectare demand of water to be zero. 
Our model of a competitively exploited aquifer can be seen as a special case of a predator-prey system, where, obviously, groundwater resembles the prey and the transformed hectares are predators. It can be demonstrated (Ibañez et al., 2004) that any intersection of $U^{R}$ and $U^{U}$ constitutes a steady state of the system, given that it always satisfies the conditions for this first established by Rosenzweig and MacArthur (1963).

As an example of time trajectory, consider an oasis initially managed in a traditional way, i.e. only employing water coming from the natural discharge of the aquifer, which gradually shifts to a competitive pumping-based system of production. This trajectory would start at the point $(\mathrm{me}, 0)$ of the phase plane and end at the steady state $\left(R^{*}, U^{*}\right)$ (Fig. 2, dashed arrow). The time employed in this process of transformation will vary for each particular case, and the transient period could show some instability, with the presence of damped oscillations. No general prediction can be made for the number of transformed hectares in the final state; they could in fact be less than the number of hectares that were irrigated under the traditional system. The only sure thing about the final state of the modelled system is that it will be established at a lower stock of groundwater than the virgin value 'rne'. This is due to the development of pumping, a flow of consumption which was absent or negligible in the initial oasis.

\section{The drivers of aquifer overexploitation}

First of all, it should be stressed that, for any given aquifer, the finding that the rate of net recharge (i.e. recharge less natural discharge) is less than the rate of consumption (i.e. total pumping less water returning to the aquifer) is a necessary but not a sufficient indicator of overexploitation. As explained at the end of last section, the development of pumping implies that the stock of groundwater at steady state will necessarily be less than it was originally. And for such a stock's decrease to occur, net pumping necessarily has to be greater than net recharge during the transient period.

The key question for assessing the risk of long-term aquifer overexploitation, then, is to determine what the values of both the stock and the hectares at which the disequilibrium between rates will stop are or, equivalently, what the position of the steady state point $\left(R^{*}, U^{*}\right)$ is. Overexploitation will happen if this final state is to the left of some critical (assumed known) value of the stock, that is to say, if $R^{*}$ is to the left of the axis $R=$ rcr in the phase plane (see Fig. 2). 'rcr' could be, for example, the volume of water needed to avoid unacceptable drawdown in particular wells. Of course, $\mathrm{rcr}=0$ means that long-term overexploitation occurs when the stock of groundwater is depleted.

Simply by looking at Fig. 2, we find that a necessary but not sufficient condition for overexploitation is

$\mathrm{rp}_{0}<\mathrm{rcr}$

That is, for the long-term overexploitation of an aquifer it is necessary but not sufficient that the stock of groundwater making the per hectare average profit to cancel out be lesser than the critical stock 'rcr'. Given Eq. (3.5), condition (4.1) also implies that $\mathrm{rrf}<\mathrm{rcr}$.

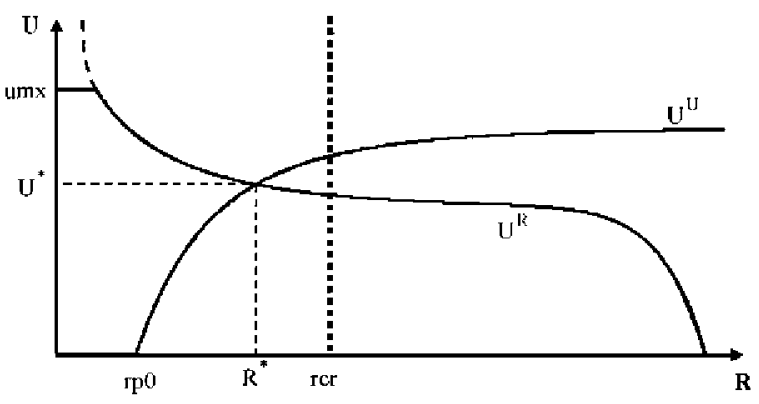

Fig. 3 - Isoclines in a case of long-term overexploitation.

Let $U^{R}(\mathrm{rcr})$ and $U^{U}(\mathrm{rcr})$ be the intersection points of $U^{R}$ and $U^{U}$ with $R=$ rcr, respectively (Fig. 3). The necessary and sufficient condition for the long-term overexploitation of the modelled aquifer is

$\mathrm{U}^{\mathrm{R}}(\mathrm{rcr})<\mathrm{U}^{\mathrm{U}}(\mathrm{rcr})$

It is unfeasible to get the mathematical expressions of $\mathrm{rp}_{0}$, $U^{R}(\mathrm{rcr})$ and $U^{U}(\mathrm{rcr})$, but their values for any applied case can always be obtained by numerical simulation if the involved parameters are known.

Fig. 4 shows the results of a sensitivity analysis of the position of $\left(R^{*}, U^{*}\right)$ under ceteris paribus conditions. The solid lines indicate the original isoclines and the dotted lines indicate the isoclines after altering the parameters. In all cases, the axis $R=r c r$ has been positioned ad hoc to show the shift of the steady state from a sustainable to a long-term overexploited situation.

Note that for the cases a-d the value of rpo stands still. This means that it would be sufficient to check that Eq. (4.1) does not hold to expect the long-term sustainability of an aquifer facing any of the parameter variations showed in those graphs.

of course, many of the model parameters actually vary in time. This means that the position of the final steady-state for a given pumped aquifer also continuously changes in time. In this way, to make a particular assessment of condition (4.2) to a real-world case either representative time parameter values (e.g. averages, modes or medians) should be used or, much better, the assessment should be repeated at interval of years. In doing so, the overall long-term sustainability of a process of agricultural transformation could be foreseen using Eq. (4.2).

\section{The case of Mediterranean aquifers}

Many studies assured, under the umbrella of dependency theory, that capital injection from western into underdeveloped countries tended to depress local economies. Common assumptions were that remittances substituted agricultural production that only survived as a sentimental or ritual activity and that they were diluted in the purchase of luxury goods and other investments rated as non-productive. However, recent studies have shown that remittances and knowledge provided by emigrants are being invested both in the modernisation of agriculture and other economic activities and that, particularly, this socioeconomic activity is triggering 
(a)

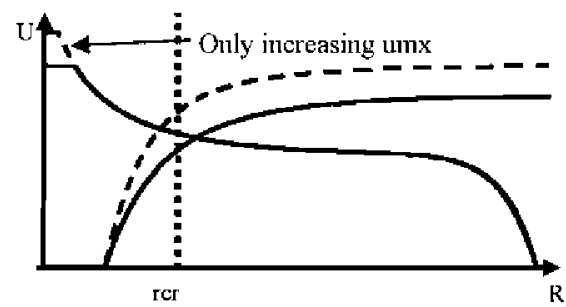

(c)

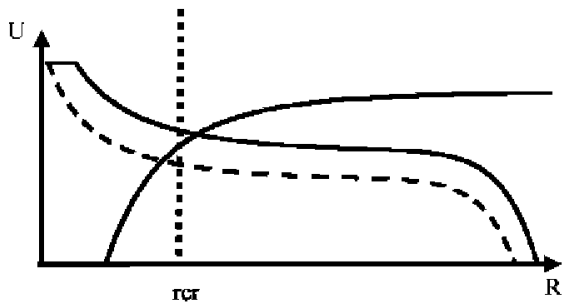

(e)

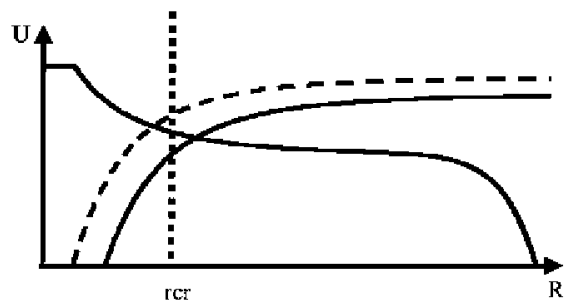

(g)

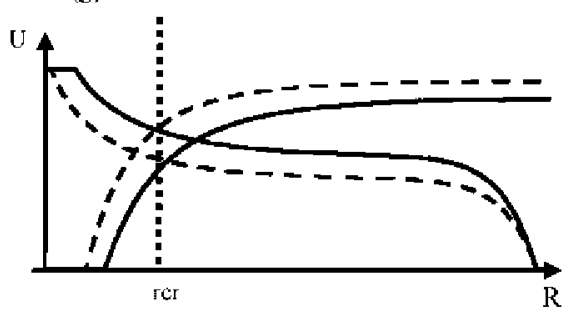

(b)

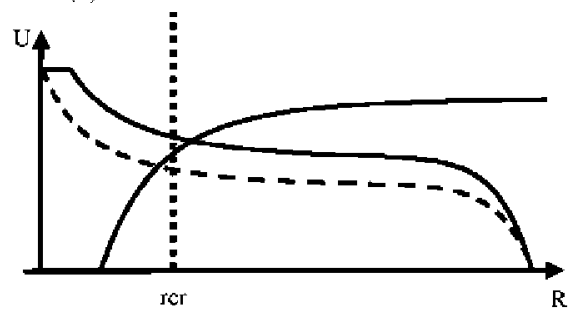

(d)

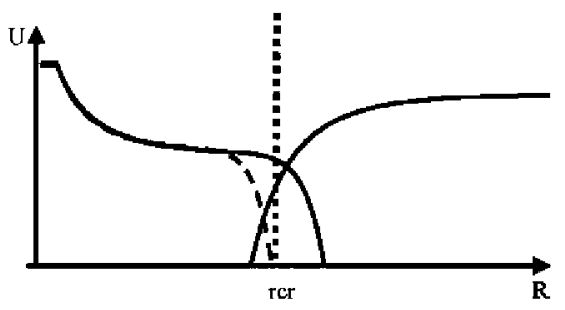

(f)

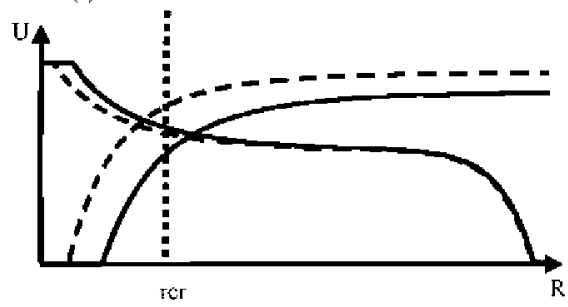

(h)

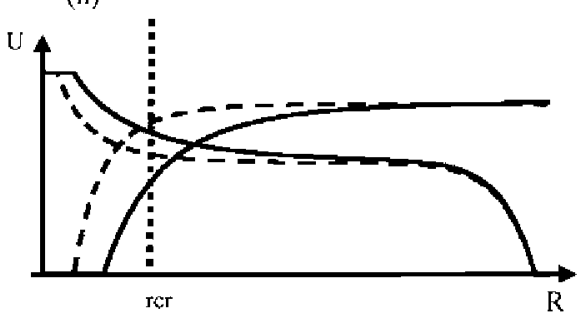

Fig. 4 - Sensitivity analysis of the steady state under ceteris paribus conditions. The solid lines correspond to the original isoclines (sustainable system); the dotted lines denote isoclines after the indicated parameter variations (overexploited system). (a) Decreasing aoc, increasing umx; (b) decreasing rfc; (c) decreasing rec; (d) decreasing rmx or dp2, increasing dp1; (e) decreasing fcu; (f) increasing prq tch; (g) increasing eqx; (h) decreasing pcr or cro.

changes in traditional water management in Maghreb's oases (Puigdefabregas and Mendizabal, 2004; De Haas, 2001).

The model proposed earlier is used in this section as a means for discussing what impact the exchange of both capital and labour between Mediterranean countries can be expected to have on sustainable groundwater management. In this way, we assess the expected long-term impacts of (i) the current development of pumping in Maghreb's oases associated to remittances and (ii) the incoming of cheap labour force to irrigated agriculture in south-eastern Spain.

Then, suppose that the model is firstly referred to some generic aquifer of a Maghreb oasis. Remittances can be considered to affect four of the model's parameters: the maximum number of irrigable hectares, 'umx'; the technology-related factor of production, 'tch'; the fraction of pumped water that percolates back into the aquifer, 'rfc'; and the average per hectare opportunity cost, 'aoc'.

External capital and knowledge of appropriate techniques are likely to result in investments in sanding and terracing. This means that arable lands can be extended by transforming terrains outside the oases into agricultural lands but with access to groundwater. Evidence of this has been found, for example, in Todgha valley (De Haas, 2001). The parameter that represents the potential arable land in the proposed model is 'umx'. As Fig. 4a illustrates, an increase in 'umx' leads to a decrease in the stock of groundwater at equilibrium, which could potentially be set under the critical value 'rcr'. Therefore, remittances would favour aquifer overexploitation in the Maghreb by increasing the arable land. 
However, some fraction of remittances is simultaneously invested in alternative businesses to agriculture so that the overall economy of the Maghreb countries is being developed. This means that farmers' opportunity cost, and, therefore, also 'aoc', are growing. Going back to Fig. 4a, we find that an increase in 'aoc' implies an increase in the stock of groundwater at equilibrium. Therefore, remittances would favour the sustainability of the Maghreb's aquifers by increasing farmers' opportunity cost.

Fig. 4a also shows that the qualitative effect on equilibrium of increasing 'aoc' is exactly the opposite to that of increasing 'umx'. Thus, the two effects of remittances mentioned so far are expected to counterbalance each other in some way.

Thirdly, remittances also favour investment in highly efficient irrigation systems to enhance productivity, that is, to get more production per volume of water employed for irrigation. This means that remittances increase 'tch' and decrease ' $r f c$ '. Figs. $4 \mathrm{~b}$ and $4 \mathrm{f}$ show that both of those shifts imply reducing the stock of water at equilibrium, which could potentially be set under 'rcr'. Hence, remittances would also favour aquifer overexploitation by increasing the investment in irrigation technology (Kendy, 2003, also finds possible negative effects of technology on ground water sustainability).

The question is, then, can the effect of remittances on 'aoc', favouring sustainability, be expected to exceed the sum of the overexploiting effects of remittances through 'umx', 'rfc' and 'tch'? In other words, could remittances increase alternative incomes for farmers enough to make them compete with the expected incomes from intensive agriculture? Or, could an investor easily find capital yields in the Maghreb's economy comparable to earnings from intensive agriculture? An affirmative response to all of these questions seems very unlikely indeed, especially after knowing the Spanish case. As a result, remittances should be taken as potential drivers of aquifer overexploitation in Maghreb oases and, therefore, some form of agreement between farmers and some common rules of groundwater exploitation should be favoured there as soon as possible.

Now consider the case of a competitively exploited aquifer for intensive agriculture in south-eastern Spain. Here, the demand for non-specialized labour attracts lots of immigrants which are bringing down wages for agricultural labourers in Spain.

Let us take our model again now referred to a Spanish aquifer. The decrease in wages due to the high supply of cheap labour reduces the fixed cost parameter, 'fcu'. As expected, and as Fig. 4e shows, the drop in 'fcu' favours resource overexploitation: the stock of groundwater in equilibrium could potentially fall below 'rcr'. Therefore, low wages for immigrant agricultural labour would favour aquifer overexploitation in Spain adding to their social effects undesirable environmental consequences. Suitable minimum wages should be assured for this labour.

Now let us suppose that some fraction of the labour for intensive agriculture in southern Spain comes from Maghreb's oases. This seems likely because in doing so they will learn about irrigated agricultural systems and will earn some money that could be invested in transforming their agricultural production systems. If this were the case, the processes of exploitation of Mediterranean aquifers for irrigation would be linked to one another and such links would be reinforcing groundwater overexploitation on both sides of the Mediterranean Sea.

Be this possibility true or not, it is important knowing how to foresee groundwater overexploitation in aquifers supporting processes of transformation from traditional to highly intensive agriculture. This paper has proposed and exemplified a general procedure for helping such early-stage monitoring in particular aquifers. Briefly, it consists in (i) formulating a theoretical dynamic model of the relationship between the irrigated hectares and the aquifer; (ii) deducing the long-term equilibrium of the model; (iii) establishing the formal conditions for that equilibrium to mean overexploitation in the long term; and (iv) assessing if these conditions hold for the particular case by quantifying the involved parameters. In this way, if the initial theoretical model is adapted to the numerical information available, the risk of overexploitation could be grossly but easily monitored periodically. Moreover, the procedure is an example of how different matters, characteristic of ecology, economics, ground water dynamics and sustainable development, can be explicit and formally put to work jointly.

\section{REFEREN CES}

Bredehoeft, J.D., 2002. The water budget myth revisited: why hydrogeologist model. Ground Water 40 (4), 340-345.

De Haas, H. (Ed.), 2001. Migration, agricultural transformations and natural resource exploitation in the oases of Morocco and Tunisia. Final scientific report IMAROM research project (Interaction between Migration, Land and Water Management and Resource Exploitation in the Oases of the Maghreb). University of Amsterdam, Amsterdam.

Edelstein-Keshet, L., 1988. Mathematical Models in Biology. The Random House, New York.

Ibañez, J., Martínez, S., Martínez, J., 2004. Competitive and optimal control strategies for groundwater pumping by agricultural production units. Water Resource Research 40 (3), W03402, doi:10.1029/2003WR002486

Ibañez, J., Martínez, J, Puigdefábregas, J., 2008. Assessing desertification risk using system stability condition analysis. Ecological Modelling 213 (2), 180-190.

Kendy, E., 2003. The false promise of sustainable pumping rates. Ground Water 41 (1), 2-4.

Martínez Vicente, S., Ibáñez, J., García-Dory, M.A., 1989. Dalagua: Un Modelo de Simulación para la Comarca de Dalias. In: Junta de Andalucía (Ed.), Recursos naturales y crecimiento económico en el Campo de Dalías. Monografías de Economía y medio Ambiente, 2, pp. 179-203, Seville.

Puigdefabregas, J., Mendizabal, T., 2004. Prospects of desertification impacts in Western Europe. In: Marquina, A. (Ed.), Environmental Challenges in the Mediterranean 2000-2050. Kluwer Academic Publisher, Netherlands, pp. 155-174.

Rosenzweig, M.L., MacArthur, R.H., 1963. Graphical representation and stability conditions of predator-prey interactions. American Naturalist 97, 209-223.

Sterman, J.D., 2000. Business Dynamics. Irwin/MacGraw-Hill, Boston.

Tout, D., 1990. The horticulture industry of Almeria Province, Spain. The Geographical Journal 156 (3), 304-312. 